\title{
Programmed cell death protein 1 expression is an independent prognostic factor in gastric cancer after curative resection
}

\author{
Shohei Eto ${ }^{1} \cdot$ Kozo Yoshikawa $^{1} \cdot$ Masaaki Nishi $^{1} \cdot$ Jun Higashijima $^{1} \cdot$ \\ Takuya Tokunaga $^{1} \cdot$ Toshihiro Nakao $^{1} \cdot$ Hideya Kashihara ${ }^{1}$ Chie Takasu ${ }^{1}$. \\ Takashi Iwata $^{1} \cdot$ Mitsuo Shimada $^{1}$
}

Received: 2 April 2015/Accepted: 10 July 2015/Published online: 26 July 2015

(C) The International Gastric Cancer Association and The Japanese Gastric Cancer Association 2015

\begin{abstract}
Background Programmed cell death protein 1 (PD-1) and its ligand PD-L1 downregulate $\mathrm{T}$ cell activation and are related to immune tolerance. The aim of this study was to clarify the significance of PD-1 and PD-L1 expression and to analyze the relationships among PD-1, PD-L1, and Foxp3 expression in gastric cancer.

Methods A total of 105 patients who underwent curative gastrectomy for stage II/III gastric cancer were included in this study. PD-1, PD-L1, and Foxp3 expression were examined by immunohistochemistry and related to prognostic factors by univariate and multivariate analyses.

Results PD-1 expression was correlated with both PD-L1 and Foxp3 expression. Disease-free survival (DFS) was significantly poorer in PD-1-positive patients than in PD-1negative patients (3-year DFS, $36.1 \%$ vs. $64.7 \%$, respectively; $p<0.05)$. Overall survival also tended to be poorer in PD-L1-positive patients than in PD-L1-negative patients. Univariate analysis identified sex, $\mathrm{T}$ factor, lymphatic invasion, and PD-1 positivity as significant predictors of poor DFS. Multivariate analysis confirmed male sex, lymphatic invasion, and positive PD-1 expression as independent prognostic indicators.

Conclusions PD-1 expression is associated with a poor prognosis and is correlated with PD-L1 and Foxp3 expression in patients with gastric cancer.
\end{abstract}

Kozo Yoshikawa

yoshikawa.kozo@tokushima-u.ac.jp

1 Department of Surgery, Graduate School of Medical Sciences, University of Tokushima, 3-18-15 Kuramoto-cho, Tokushima 770-8503, Japan
Keywords Gastric cancer - Programmed cell death 1 . Programmed cell death ligand 1

\begin{tabular}{|c|c|}
\hline \multicolumn{2}{|c|}{ Abbreviations } \\
\hline DFS & Disease-free survival \\
\hline OS & Overall survival \\
\hline PD-1 & Programmed cell death protein 1 \\
\hline PD-L1 & Programmed cell death ligand 1 \\
\hline Tregs & Regulatory $\mathrm{T}$ cells \\
\hline
\end{tabular}

\section{Introduction}

Dysfunction of the immune system in cancer patients allows cancer cells to avoid immune surveillance [1, 2]. Immune tolerance involves loss of cancer antigens, alterations in cell death receptor signaling, lack of $\mathrm{T}$ cells or antibodies, and exhausted $\mathrm{T}$ cells, intratumoral monocytes, and regulatory $\mathrm{T}$ cells (Tregs) [1].

Programmed cell death protein 1 (PD-1) is a CD28 family member first reported in 1992. PD-1 is expressed on activated $\mathrm{T}$ cells, $\mathrm{B}$ cells, and myeloid cells, and its expression is enhanced by classic programmed cell death [3]. PD-1 and its ligand, programmed cell death ligand 1 (PD-L1), interact to downregulate the activation of T cells in autoimmune disease, chronic infection, and cancer [4, 5]. PD-1 is also known to activate the transcription factor Foxp3, and is correlated with Foxp3 expression [6]. Several recent reports have demonstrated a correlation between PD-1 expression and prognosis in cancer patients. However, little is known about the significance of the relationship between PD-1 and PD-L1 expression in gastric cancer. 
The aim of this study was to clarify the significance of PD-1 and PD-L1 expression and to analyze the relationships among PD-1, PD-L1, and Foxp3 expression in patients with gastric cancer.

\section{Patients and methods}

\section{Patients}

A total of 105 patients who had undergone surgical resection for stage II/III gastric cancer at Tokushima University Hospital between 2006 and 2012 were included in this study (42 patients with stage II gastric cancer and 63 patients with stage III gastric cancer). There were 75 men and 30 women, with a mean age of 67.8 years (range 38-95 years). Thirty-nine patients $(37.1 \%)$ underwent total gastrectomy and 66 patients $(62.9 \%)$ underwent distal gastrectomy. The mean follow-up period was 34 months (range 7-87 months). Seventy-three patients underwent adjuvant chemotherapy. Factors were defined according to the 14th edition of the Japanese Classification of Gastric Carcinoma [7]. This study was authorized in advance by the Institutional Review Board of the University of Tokushima Graduate School of Medical Science (Institutional Review Board number 1517), and all patients provided written informed consent.

\section{Immunohistochemistry}

Tissue samples were formalin fixed and paraffin embedded. Serial sections were cut at $5 \mu \mathrm{m}$, dewaxed, deparaffinized in xylene, and rehydrated through a series of graded alcohols. Samples were boiled for $20 \mathrm{~min}$ in a microwave oven in citrate buffer $(\mathrm{pH}$ 6.0) for antigen retrieval. Endogenous peroxidases were blocked with $0.3 \%$ hydrogen peroxidase for $30 \mathrm{~min}$, followed by incubation in $5 \%$ goat serum for $60 \mathrm{~min}$ to prevent nonspecific antigen binding. The slides were then incubated with primary antibodies overnight at $4{ }^{\circ} \mathrm{C}$. The following primary antibodies and dilutions were used: mouse monoclonal antibody for PD-1 (AF1086, 1:40; R\&D Systems, Minneapolis, MN, USA), rabbit monoclonal antibody for PD-L1 (ab174838, 1:100; Abcam, Cambridge, UK), and mouse monoclonal antibody for Foxp3 (ab20034, 1:100; Abcam). Secondary antibody binding was detected with Histofine SAB-PO (Nichirei) for PD-1 and EnVision Dual Link System-HRP (DAKO) for PD-L1 and Foxp3. A secondary peroxidase-labeled polymer conjugated to goat anti-mouse immunoglobulins was applied for $60 \mathrm{~min}$. The sections were developed in 3,3-diaminobenzidine and were counterstained with Mayer's hematoxylin. Each slide was dehydrated through graded alcohols and covered with a coverslip. The presence of positive cells on each slide was determined by a pathologist in a blinded manner. PD-1 positivity was recorded if more than $40 \% \mathrm{~T}$ cells were stained in a $\times 400$ high-power field at the center of the tumor (Fig. 1, panel A) [8]. Twenty-eight patients (27\%) were PD-1 positive.

PD-L1 expression was predominantly located in the cytoplasm, with some nuclear membrane localization at the invasive front of the tumor. Staining intensity was graded as follows: 0 for no staining, $1+$ for weak staining, $2+$ for moderate staining, and $3+$ for strong staining. Distribution was graded according to the percentage of PD-L1-positive cancer cells and then divided into quartiles as follows: no staining, 0-5\% staining; $1+, \quad 6-25 \%$ staining; 2+, 26-50\% staining; $3+, \quad 51-75 \%$ staining; and $4+$, $76-100 \%$ staining. A total score of more than $3+$ was defined as PD-L1-positive expression (Fig. 1, panel B) [9]. Twenty-six patients (25\%) were PD-L1 positive.

We recorded Foxp3 positivity by counting more than ten Foxp3-stained $\mathrm{T}$ cells in the cancer tissue at $\times 200$ highpower field at the center of the tumor (Fig. 1, panel C) [10, 11]. Thirty-four patients (32\%) were Foxp3 positive.

\section{Statistical analysis}

All statistical analyses were performed with JMP 8.0.1 (SAS, Cary, NC, USA). Continuous variables were compared by Mann-Whitney $U$ tests, and categorical data were compared by $\chi^{2}$ tests. Survival curves were calculated by the Kaplan-Meier method and compared by log-rank tests. The prognostic potentials of the parameters were analyzed by univariate analysis. Relative risk and $95 \%$ confidence intervals were estimated with the Cox proportional hazards model with stepwise forward selection. Statistical significance was defined as $p<0.05$.

\section{Results}

The characteristics of the PD-1-positive and PD-1-negative groups are shown in Table 1. In terms of clinicopathological variables, PD-1 expression was positively correlated with PD-L1 expression $(p<0.001)$ and Foxp3 expression $(p=0.002)$ (Fig. 2a, b). PD-L1 expression was correlated with the depth of the tumor $(p=0.032)$ (Table 2$)$ and Foxp3 expression $(p<0.001)$ (Fig. 2c).

Overall survival (OS) rates were similar in patients with and without PD-1 expression (3-year OS rate, $69.0 \%$ for PD-1-positive patients vs. $81.8 \%$ for PD-1-negative patients; $p=0.55$ ) (Fig. 3a). However, disease-free survival (DFS) was significantly poorer in the PD-1-positive group than in the PD-1 negative group (3-year DFS rate, $36.1 \%$ vs. $64.7 \%$, respectively; $p<0.05$ ) (Fig. 3b). OS 
Fig. 1 Immunohistochemistry: a programmed cell death protein $1(P D-1)$-positive expression in cancer tissue; $b$ strong programmed cell death ligand 1 $(P D-L 1)$ staining and $70 \%$ stained cells; c Foxp3-positive expression in cancer tissue
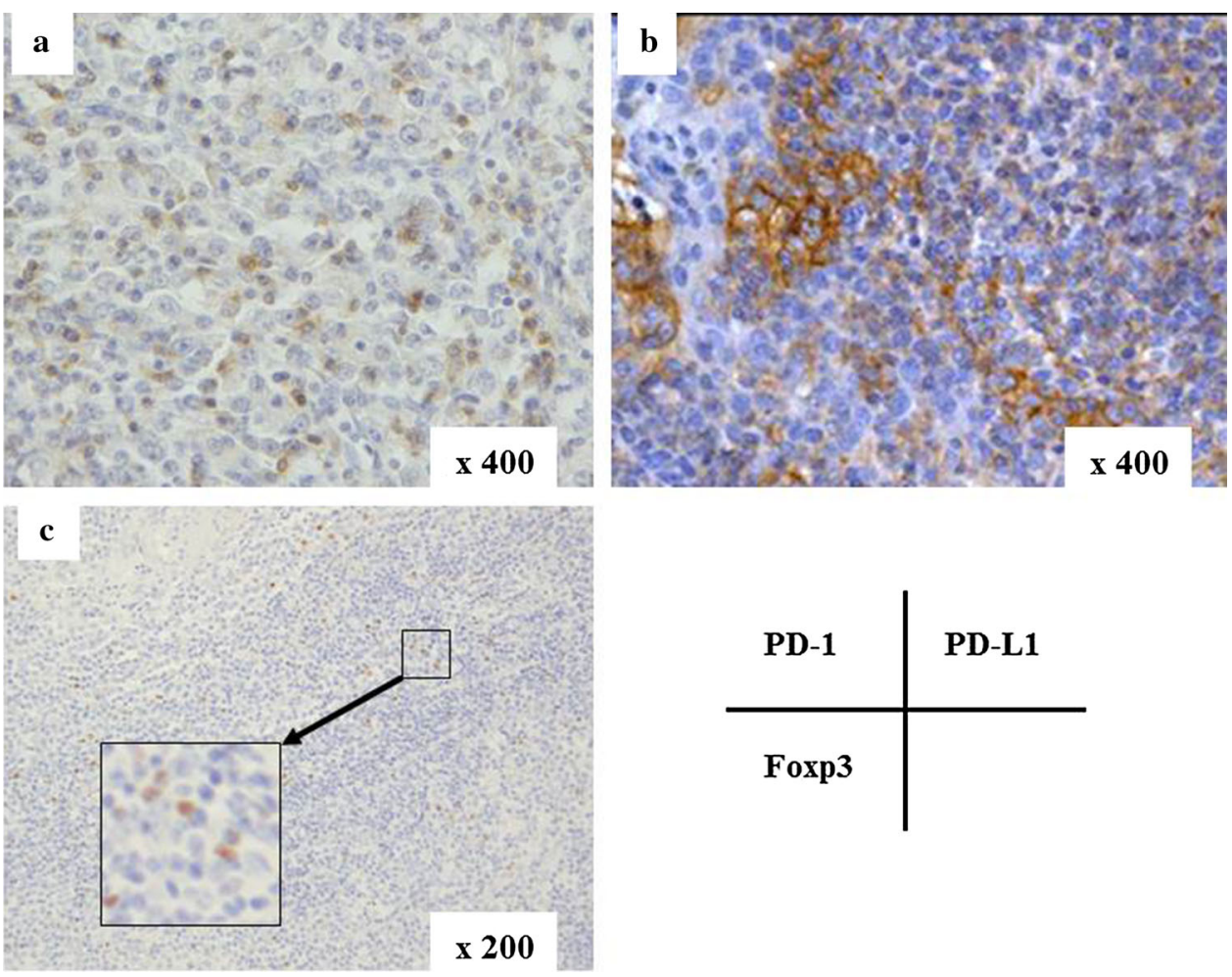

Table 1 Characteristics of programmed cell death protein 1 (PD-1)-positive and PD-1negative patients

\begin{tabular}{llll}
\hline Variables & Positive $(n=28)$ & Negative $(n=77)$ & $p$ \\
\hline Age & $67 \pm 14$ years & $68 \pm 11$ years & 0.683 \\
Sex (male/female) & $18 / 10$ & $57 / 20$ & 0.331 \\
Differentiation (differentiated/undifferentiated) & $13 / 15$ & $39 / 38$ & 0.704 \\
Depth of tumor invasion (T1, T2/T3, T4) & $18 / 10$ & $56 / 21$ & 0.402 \\
Lymph mode metastasis (-/+) & $7 / 21$ & $15 / 62$ & 0.538 \\
Stage (II/III) & $10 / 18$ & $32 / 45$ & 0.588 \\
Venous invasion (-/+) & $9 / 19$ & $23 / 54$ & 0.324 \\
Lymphatic invasion (-/+) & $16 / 12$ & $52 / 25$ & 0.331 \\
Adjuvant chemotherapy (-/+) & $7 / 21$ & $25 / 52$ & 0.462 \\
\hline
\end{tabular}

tended to be lower in the PD-L1-positive group than in the PD-L1-negative group, although the difference was not significant (3-year OS rate, $65.8 \%$ vs. $81.8 \%$, respectively; $p=0.08$ ) (Fig. 3c). However, there was no significant difference between the two groups in terms of DFS (3-year DFS rate, $41.9 \%$ vs. $62.4 \%$, respectively; $p=0.18$ ) (Fig. 3d). In addition, the group with both PD-1positive and PD-L1-positive expression $(n=16)$ had poorer OS than the group with double negative expression (3-year OS rate, $63.8 \%$ vs. $82.8 \%$, respectively; $p=0.124$ ) (Fig. 3e), and significantly poorer DFS (3-year DFS rate, $49.2 \%$ vs. $68.1 \%$, respectively; $p=0.049$ ) (Fig. 3f).

Univariate analysis identified $\operatorname{sex}(p=0.035), \mathrm{T}$ factor $(p=0.046)$, lymphatic invasion $(p=0.007)$, and PD-1 expression ( $p=0.022$ ) as significant prognostic factors for DFS (Table 3). Multivariate analysis confirmed male sex, lymphatic invasion, and PD-1 expression as independent risk factors for recurrence (relative risks of 3.47, 2.66, and 2.43, respectively) (Table 4).

\section{Discussion}

The results of this study demonstrated that PD-1 expression was a poor prognostic factor in terms of DFS, and was correlated with PD-L1 and Foxp3 expression in patients with stage II/III gastric cancer after curative resection. This is the first report to clarify the relationships among PD-1, PD-L1, and Foxp3 expression in gastric cancer. 
Fig. 2 Correlation between a programmed cell death protein $1(P D-1)$ and programmed cell death ligand $1(P D-L 1)$, b PD-1 and Foxp3, and $\mathbf{c}$ PD-L1 and Foxp3
Table 2 Characteristics of programmed cell death ligand 1 (PD-L1)-positive and PD-L1negative patients
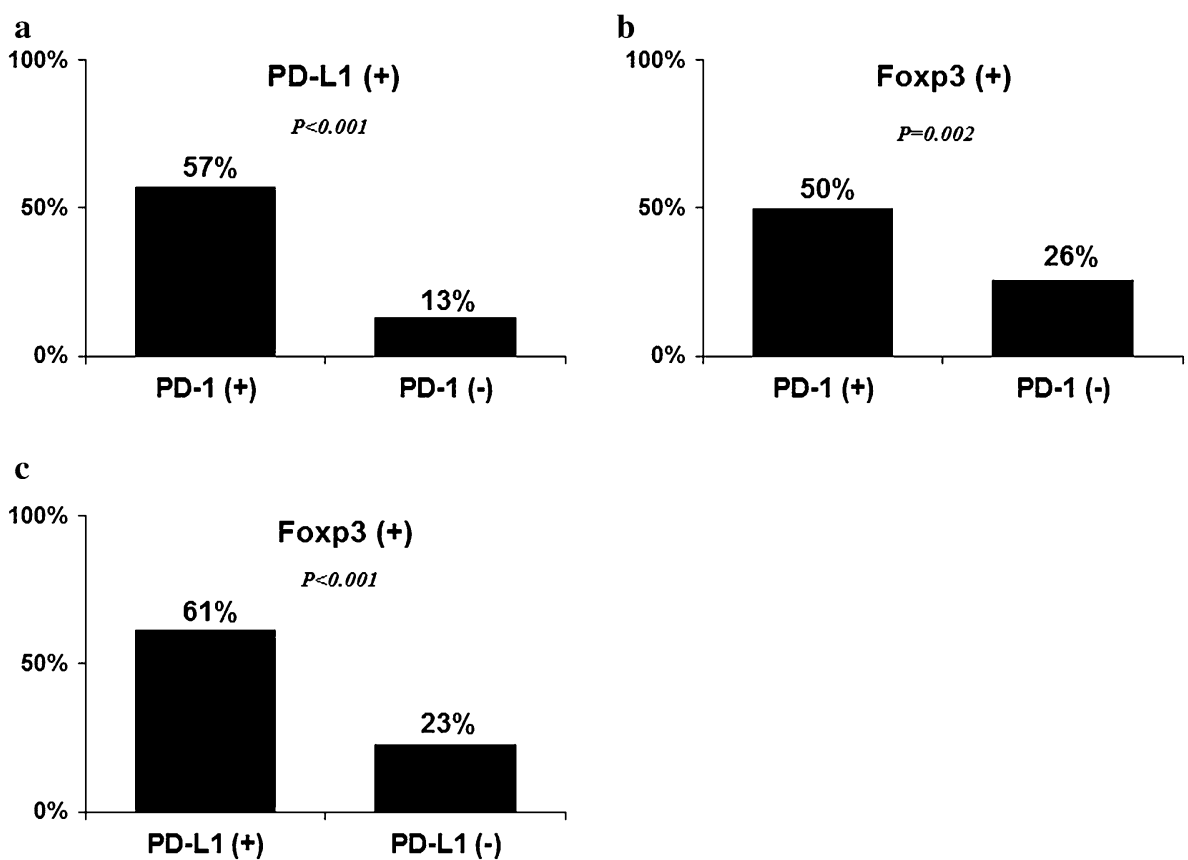

\begin{tabular}{llll}
\hline Variables & Positive $(n=26)$ & Negative $(n=79)$ & $p$ \\
\hline Age & $67 \pm 14$ years & $68 \pm 12$ years & 0.949 \\
Sex (male/female) & $17 / 9$ & $58 / 21$ & 0.427 \\
Differentiation (differentiated/undifferentiated) & $13 / 13$ & $40 / 39$ & 0.963 \\
Depth of tumor invasion (T1, T2/T3, T4) & $12 / 14$ & $60 / 19$ & 0.032 \\
Lymph mode metastasis (-/+) & $6 / 20$ & $16 / 63$ & 0.761 \\
Stage (II/III) & $9 / 17$ & $33 / 46$ & 0.518 \\
Venous invasion (-/+) & $8 / 18$ & $24 / 55$ & 0.972 \\
Lymphatic invasion (-/+) & $17 / 9$ & $51 / 28$ & 0.928 \\
Adjuvant chemotherapy (-/+) & $8 / 18$ & $24 / 55$ & 0.970 \\
\hline
\end{tabular}

PD-1 and PD-L1 play important roles in the regulation of the immune system and the maintenance of peripheral tolerance through $\mathrm{T}$ cell activation and tolerance $[9,12]$. In a systematic review, PD-1 or PD-L1 expression status was a significant prognostic factor in epithelial-originated malignancies, and the mechanism of this immune tolerance was discussed [13]. This report showed that PD-1 is induced on $\mathrm{T}$ cells in response to inflammatory stimuli, and tumor cells can express PD-L1 to inhibit T-cell-mediated antitumor immunity since PD-L1 can recognize and bind PD-1 on tumor-infiltrating lymphocytes [13].

In gastric cancer, few studies have examined PD-1 and PD-L1, and their roles remain controversial [14]. One report showed that PD-L1-positive expression was associated with poor prognosis in advanced gastric adenocarcinoma, whereas another proposed that increased PD-1 expression on $\mathrm{CD}^{+}{ }^{+}$and $\mathrm{CD}^{+}{ }^{+} \mathrm{T}$ cells was involved in immune evasion [15]. Another study examined PD-L1, cytotoxic
T-lymphocyte-associated antigen 4, and indolamine 2,3dioxygenase expression by immunohistochemistry, and showed that PD-L1 positivity and a high-CD3 microenvironment were related to favorable survival outcomes [14].

In our experiments, PD-1 was positively correlated with expression of the transcription factor Foxp3, which is widely known to be involved in the development and function of Tregs [16]. Tregs have shown significant correlations with cancer progression and metastasis by inhibiting the $\mathrm{T}$ cell response via membrane-bound transforming growth factor $\beta_{1}$ [17]. PD-1 was partly expressed on Tregs and was identified as the key regulator of immune tolerance [18]. PD-1, PD-L1, and Foxp3 have previously been found to be associated with disease progression in breast cancer via immunosuppressive subsets of $\mathrm{T}$ cells in the cancer microenvironment [19]. These findings suggest that PD-1-positive $\mathrm{T}$ cells might induce Tregs via transforming growth factor $\beta_{1}$ in gastric cancer. 
Fig. 3 Kaplan-Meier analysis of overall survival and diseasefree survival for programmed cell death protein $1(P D-1)$ expression $(\mathbf{a}, \mathbf{b})$ and programmed cell death ligand 1 $(P D-L 1)$ expression $(\mathbf{c}, \mathbf{d})$ after curative resection in patients with gastric cancer. Survival for double PD-1 and PD-L1 expression $(\mathbf{e}, \mathbf{f})$ $\mathbf{a}$

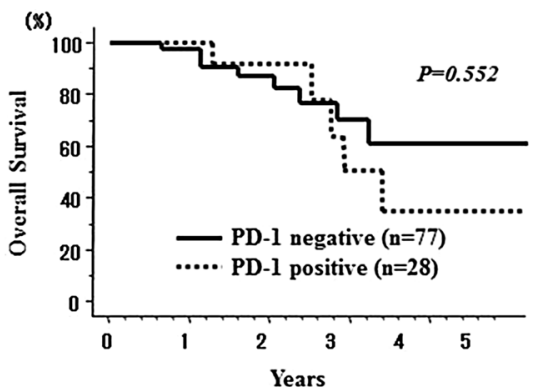

c

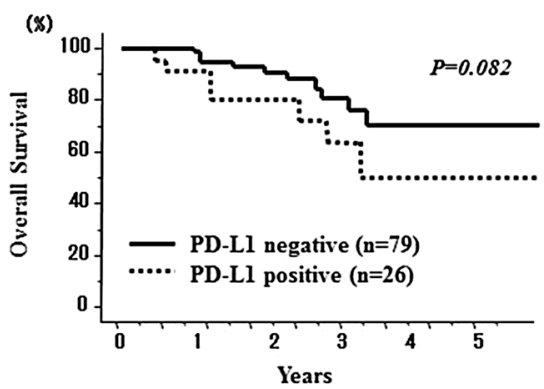

e

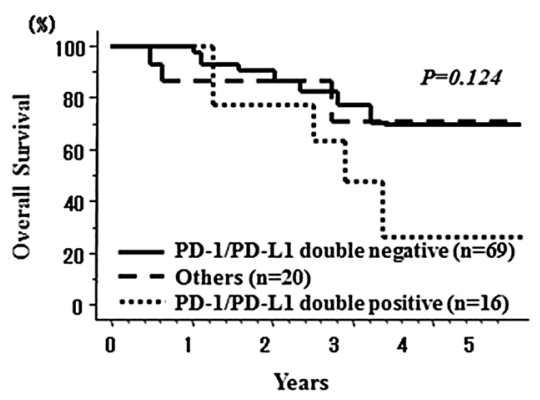

b

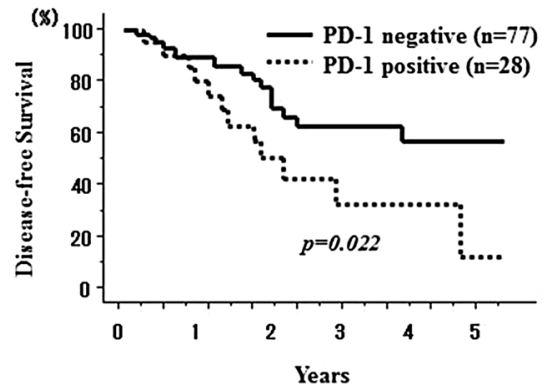

d

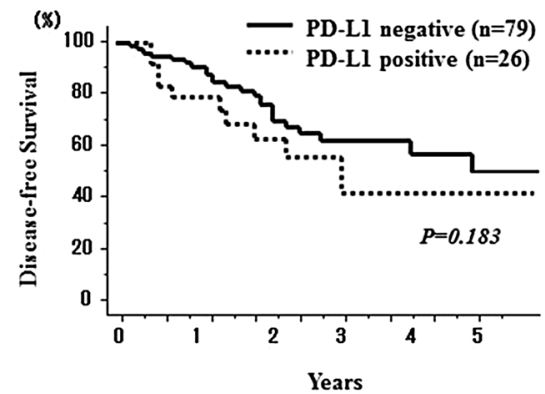

f

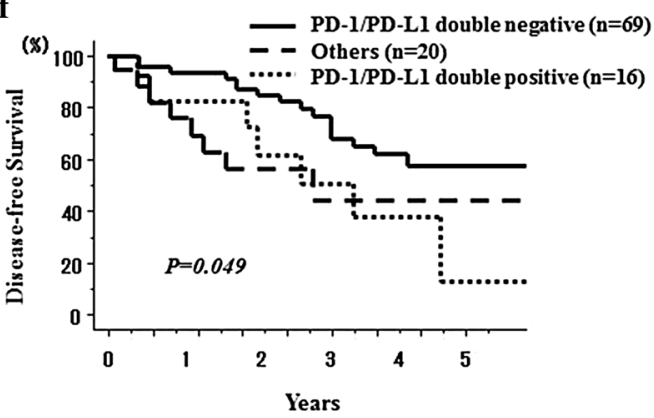

Table 3 Univariate analysis of prognostic factors for disease-free survival $(D F S)$

\begin{tabular}{lll}
\hline Variables & 3-year DFS rate $(\%)$ & $p$ \\
\hline Age (<70 years/ $\geq 70$ years) & $64.0 / 47.8$ & 0.146 \\
Sex (male/female) & $50.3 / 70.2$ & 0.035 \\
Diffferentiation (poor/good) & $55.9 / 57.4$ & 0.860 \\
T3, T4 (-/+) & $65.1 / 35.6$ & 0.046 \\
N1-N3 (-/+) & $55.1 / 56.4$ & 0.870 \\
Stage III (-/+) & $62.5 / 52.5$ & 0.430 \\
Venous invasion (-/+) & $74.9 / 46.6$ & 0.051 \\
Lymphatic invasion (-/+) & $69.4 / 35.0$ & 0.007 \\
PD-1 (-/+) & $64.7 / 36.1$ & 0.022 \\
PD-L1 (-/+) & $62.4 / 41.9$ & 0.181 \\
Foxp3 (-/+) & $58.6 / 52.1$ & 0.879 \\
Adjuvant chemotherapy $(-/+)$ & $64.3 / 54.1$ & 0.202 \\
\hline
\end{tabular}

$P D$-1 programmed cell death protein $1, P D-L 1$ programmed cell death ligand $1, T$ depth of tumor invasion
Table 4 Multivariate analysis of prognostic factors for disease-free survival

\begin{tabular}{llll}
\hline Variables & Relative risk & $95 \%$ CI & $p$ \\
\hline Sex (male) & 3.472 & $1.393-8.696$ & 0.008 \\
Lymphatic invasion $(+)$ & 2.661 & $1.347-5.258$ & 0.005 \\
PD-1 (+) & 2.430 & $1.217-4.852$ & 0.012 \\
\hline
\end{tabular}

$C I$ confidence interval, $P D-1$ programmed cell death protein 1

Immunotherapeutic agents targeting $\mathrm{T}$ cell immune checkpoints such as PD-1, PD-L1, cytotoxic T-lymphocyte-associated antigen 4 , and T-cell-immunoglobulin- and mucin-domain-containing molecule 3 have been investigated as potential treatments for cancer $[14,20]$. These immunotherapeutic agents have shown promising clinical efficacies for several types of cancer, including non-smallcell lung cancer, melanoma, and renal cell carcinoma [2123]. Combined treatment with cisplatin and CD137/PD-1 
monoclonal antibodies also resulted in long-term survival in mice with established TC1 lung cancer [24]. The PD-1 monoclonal antibody pembrolizumab is currently undergoing clinical trials for advanced gastric cancer (NCT02335411;https://clinicaltrials.gov/ct2/show/NCT023 35411). The present study confirmed PD-1 expression as a predictive factor for recurrence after curative gastrectomy. Immunotherapy targeting PD-1 and PD-L1 may thus be a useful adjuvant treatment in patients with gastric cancer.

In conclusion, PD-1 expression was associated with a poor prognosis and was correlated with PD-L1 and Foxp3 expression in patients with gastric cancer. The PD-1/PD-L1 pathway may be a useful new therapeutic target for the treatment of gastric cancer.

\section{Compliance with ethical standards}

Conflict of interest The authors declare that they have no conflict of interest.

Ethics statement All procedures followed were in accordance with the ethical standards of the responsible committee on human experimentation (institutional and national) and with the Helsinki Declaration of 1964 and later versions. Informed consent or substitute for it was obtained from all patients for their being included in the study.

\section{References}

1. Ahmad M, Rees RC, Ali SA. Escape from immunotherapy: possible mechanisms that influence tumor regression/progression. Cancer Immunol Immunother. 2004;53(10):844-54.

2. Rivoltini L, Canese P, Huber V, Iero M, Pilla L, Valenti R, et al. Escape strategies and reasons for failure in the interaction between tumour cells and the immune system: how can we tilt the balance towards immune-mediated cancer control? Expert Opinion Biol Ther. 2005;5(4):463-76.

3. Ishida Y, Agata Y, Shibahara K, Honjo T. Induced expression of PD-1, a novel member of the immunoglobulin gene superfamily, upon programmed cell death. EMBO J. 1992;11(11):3887-95.

4. Cao Y, Zhang L, Ritprajak P, Tsushima F, Youngnak-Piboonratanakit $\mathrm{P}$, Kamimura $\mathrm{Y}$, et al. Immunoregulatory molecule B7H1 (CD274) contributes to skin carcinogenesis. Cancer Res. 2011;71(14):4737-41.

5. Liang SC, Latchman YE, Buhlmann JE, Tomczak MF, Horwitz BH, Freeman GJ, et al. Regulation of PD-1, PD-L1, and PD-L2 expression during normal and autoimmune responses. Eur $\mathrm{J}$ Immunol. 2003;33:2706-16.

6. Ahearne MJ, Bhuller K, Hew R, Ibrahim H, Naresh K, Wagner SD. Expression of PD-1 (CD279) and FoxP3 in diffuse large B-cell lymphoma. Virchows Arch. 2014;465(3):351-8. doi:10. 1007/s00428-014-1615-5.

7. Japanese Gastric Cancer Association. Japanese classification of gastric carcinoma: 3rd English edition. Gastric Cancer. 2011;101-12. doi:10.1007/s10120-011-0041-5.

8. Muenst S, Soysal SD, Gao F, et al. The presence of programmed death 1 (PD-1)-positive tumor-infiltrating lymphocytes is associated with poor prognosis in human breast cancer. Breast Cancer Res Treat. 2013;139(3):667-76.

9. Flies DB, Chen L. The new B7s: playing a pivotal role in tumor immunity. J Immunother. 2007;30:251-60.

10. Droeser R, Zlobec I, Kilic E, Güth U, Heberer M, Spagnoli G, et al. Differential pattern and prognostic significance of CD4+, FOXP3+ and IL-17+ tumor infiltrating lymphocytes in ductal and lobular breast cancers. BMC Cancer. 2012;3(12):134.

11. Flammiger A, Weisbach L, Huland $\mathrm{H}$, et al. High tissue density of FOXP3 + T cells is associated with clinical outcome in prostate cancer. Eur J Cancer. 2013;49(6):1273-9.

12. Taube JM, Klein A, Brahmer JR, Xu H, Pan X, Kim JH, et al. Association of PD-1, PD-1 ligands, and other features of the tumor immune microenvironment with response to anti-PD-1 therapy. Clin Cancer Res. 2014;20(19):5064-74.

13. Zhang Y, Kang S, Shen J, He J, Jiang L, Wang W, et al. Prognostic significance of programmed cell death 1 (PD-1) or PD-1 ligand 1 (PD-L1) expression in epithelial-originated cancer: a meta-analysis. Medicine (Baltimore). 2015;94(6):e515. doi:10. 1097/MD.0000000000000515.

14. Kim JW, Nam KH, Ahn S-H, Park DJ, Kim H-H, Kim SH, et al. Prognostic implications of immunosuppressive protein expression in tumors as well as immune cell infiltration within the tumor microenvironment in gastric cancer. Gastric Cancer. 2014. doi:10.1007/s10120-014-0440-5.

15. Saito H, Kuroda H, Matsunaga T, Osaki T, Ikeguchi M. Increased $\mathrm{PD}-1$ expression on $\mathrm{CD} 4+$ and $\mathrm{CD} 8+\mathrm{T}$ cells is involved in immune evasion in gastric cancer. $J$ Surg Oncol. 2013;107(5):517-22.

16. Douglass S, Ali S, Meeson AP, Browell D, Kirby JA. The role of FOXP3 in the development and metastatic spread of breast cancer. Cancer Metastasis Rev. 2012;31(3-4):843-54.

17. Han Y, Yang Y, Chen Z, Jiang Z, Gu Y, Liu Y, et al. Human hepatocellular carcinoma-infiltrating $\mathrm{CD} 4{ }^{+} \mathrm{CD} 69^{+} \mathrm{Foxp}^{-}$regulatory $\mathrm{T}$ cell suppresses $\mathrm{T}$ cell response via membrane-bound TGF- $\beta 1$. J Mol Med (Berl). 2014;92(5):539-50.

18. Bodhankar S, Vandenbark AA, Offner H. Oestrogen treatment of experimental autoimmune encephalomyelitis requires $17 \beta$ oestradiol-receptor-positive $\mathrm{B}$ cells that up-regulate PD-1 on CD4+ Foxp3+ regulatory $\mathrm{T}$ cells. Immunology. 2012;137(4):282-93.

19. Sun S, Fei X, Mao Y, Wang X, Garfield DH, Huang O, et al. PD$1(+)$ immune cell infiltration inversely correlates with survival of operable breast cancer patients. Cancer Immunol Immunother. 2014;63(4):395-406.

20. Merelli B, Massi D, Cattaneo L, Mandala M. Targeting the PD1/ PD-L1 axis in melanoma: biological rationale, clinical challenges and opportunities. Crit Rev Oncol Hematol. 2014;89:140-65.

21. Topalian SL, Hodi FS, Brahmer JR, Gettinger SN, Smith DC, McDermott DF, et al. Safety, activity, and immune correlates of anti-PD-1 antibody in cancer. N Engl J Med. 2012;366(26):2443-54.

22. Brahmer JR, Tykodi SS, Chow LQ, Hwu WJ, Topalian SL, Hwu $\mathrm{P}$, et al. Safety and activity of anti-PD-L1 antibody in patients with advanced cancer. N Engl J Med. 2012;366(26):2455-65.

23. Robert C, Thomas L, Bondarenko I, O’Day S, MD JW, Garbe C, et al. Ipilimumab plus dacarbazine for previously untreated metastatic melanoma. N Engl J Med. 2011;364:2517-26.

24. Wei H, Zhao L, Li W. Combinatorial PD-1 blockade and CD137 activation has therapeutic efficacy in murine cancer models and synergizes with cisplatin. PLoS One. 2013;19;8(12):e84927. 